\title{
Relaxation of magnetic field relative to plasma density during solar flares
}

\author{
Sijie Yu${ }^{1}$, Yihua Yan ${ }^{1}$, and Baolin Tan ${ }^{1}$ \\ ${ }^{1}$ Key Laboratory of Solar Activity, National Astronomical Observatories of the Chinese \\ Academy of Sciences, Beijing 100012, China \\ email: sjyu@nao.cas.cn
}

\begin{abstract}
We investigated the variations of 74 microwave ZP structures observed by Chinese Solar Broadband Radio Spectrometer at $2.6-3.8 \mathrm{GHz}$ in 9 solar flares, found that the ratio between the plasma density scale height $L_{N}$ and the magnetic field scale height $L_{B}$ in emission source displays a tendency of decrease during the flaring process, indicates that $L_{B}$ increases faster than the $L_{N}$ during solar flares. The detailed analysis of the step-wise decrease of $L_{N} / L_{B}$ in three typical X-class flares reveals the magnetic field relaxation relative to the plasma density.
\end{abstract}

Keywords. Sun: flares — Sun: magnetic topology — Sun: radio radiation

\section{Introduction}

Zebra pattern (ZP) is an intriguing spectral structures in the solar radio emission (Elgaröy 1959; Chernov 2006), which consists of a number of almost parallel and equidistant stripes superimposed on the type IV radio burst in dynamic spectrum, providing a unique diagnostics of the magnetic field and ambient plasma around the source (Tan, et al. 2012). It is generally considered that the emission of microwave fine ZP structures requires high density and high temperature (Bastian et al. 1998), implies that the source of microwave ZP may be located very closed to the flare core region where the magnetic energy is released. From the double plasma resonance model, we may obtain the intrinsic information of the flare core region by calculating the ratio of scale lengths of plasma density and magnetic field $\left(L_{N} / L_{B}\right)$ (Kuznetsov \& Tsap 2007).

\section{Observations and Results}

Table 1 listed 74 ZPs in 9 solar flares from 1997 to 2006, whose $L_{N} / L_{B}$ can be calculated (Fig. 1). We found: (1) During the flaring processes, the ratio is mainly in the range

Table 1. The list of flare events with microwave ZPs at 2.6-3.8 GHz from 1997 to 2006

\begin{tabular}{|c|c|c|c|c|c|c|c|c|c|c|c|}
\hline \multicolumn{4}{|c|}{ Flare } & \multicolumn{8}{|c|}{$\mathbf{Z P}$} \\
\hline Event & Class & $\begin{array}{c}\text { AR } \\
\text { NOAA }\end{array}$ & $\underset{U T}{\text { SXR peak }}$ & $\begin{array}{l}\text { Start } \\
\text { UT }\end{array}$ & $\begin{array}{c}\text { End } \\
\text { UT }\end{array}$ & $\begin{array}{c}\mathbf{f} \\
\mathrm{GHz}\end{array}$ & $\begin{array}{c}\Delta f \\
\mathrm{MHz}\end{array}$ & Pol & No & $\mathrm{Ns}$ & $\mathrm{S}$ \\
\hline 09.04 .2000 & M3.1 & 8948 & $23: 42$ & $23: 35: 12$ & $23: 35: 18$ & $2.60-3.10$ & $20-40$ & STRONG R & 1 & 3 & $\diamond$ \\
\hline 29.10 .2000 & $\mathrm{C} 4.4$ & 9209 & $01: 57$ & $02: 06: 32$ & $02: 35: 05$ & $2.60-3.10$ & $65-80$ & $\mathrm{R}$ & 17 & $3-7$ & $\Delta$ \\
\hline 24.11 .2000 & $\mathrm{X} 2.0$ & 9236 & 05:02 & $04: 59: 56$ & $05: 01: 57$ & $2.60-3.80$ & $55-60$ & $L \& R$ & 4 & 3 & $\square$ \\
\hline 25.11 .2000 & M 8.2 & 9240 & $01: 31$ & $00: 59: 19$ & 01:09:30 & $2.60-3.80$ & 50 & $L \& R$ & 4 & $5-6$ & 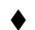 \\
\hline 19.10 .2001 & $\mathrm{X} 1.6$ & 9661 & 01:05 & $00: 51: 00$ & $01: 19: 55$ & $2.60-3.00$ & $55-75$ & $\mathrm{R}$ & 18 & $3-8$ & $x$ \\
\hline 21.04 .2002 & X1.5 & 9906 & $01: 50$ & $01: 45: 40$ & $02: 01: 45$ & $2.60-3.80$ & $30-70$ & $\mathrm{~L}$ & 10 & $10-34$ & + \\
\hline 18.11 .2003 & M3.9 & 10501 & $08: 31$ & $08: 22: 42$ & $08: 26: 50$ & $2.60-3.50$ & $30-50$ & STRONG R & 3 & $3-5$ & $\Delta$ \\
\hline 09.07 .2005 & M2.8 & 10786 & $22: 06$ & $22: 03: 16$ & $22: 04: 53$ & $2.60-3.50$ & $50-80$ & $L \& R$ & 6 & 8 & $\mathbf{\square}$ \\
\hline 13.12 .2006 & X3.4 & 10930 & $02: 40$ & $02: 22: 30$ & 03:03:00 & $2.60-3.80$ & $50-250$ & $\mathrm{R}$ & 13 & $3-6$ & $*$ \\
\hline
\end{tabular}

Notes.- Start (UT)- time of the first ZP in the event; End (UT)- time of the last ZP in the event; AR NOAAnumber of NOAA active region; SXR peak (UT)- time of soft X-ray peak; $f(\mathrm{GHz})$ - frequency range of $\mathrm{ZPs} ; \Delta \mathrm{f}$ $(\mathrm{MHz})$ - frequency separation of adjacent stripes; Pol- left- or right-handed circular polarization; No- number of ZPs; Ns- stripes number in a ZP event; Ref- reference; S- the symbols used in Figure 1 (Yu et al. 2012). 
Before the flare peak

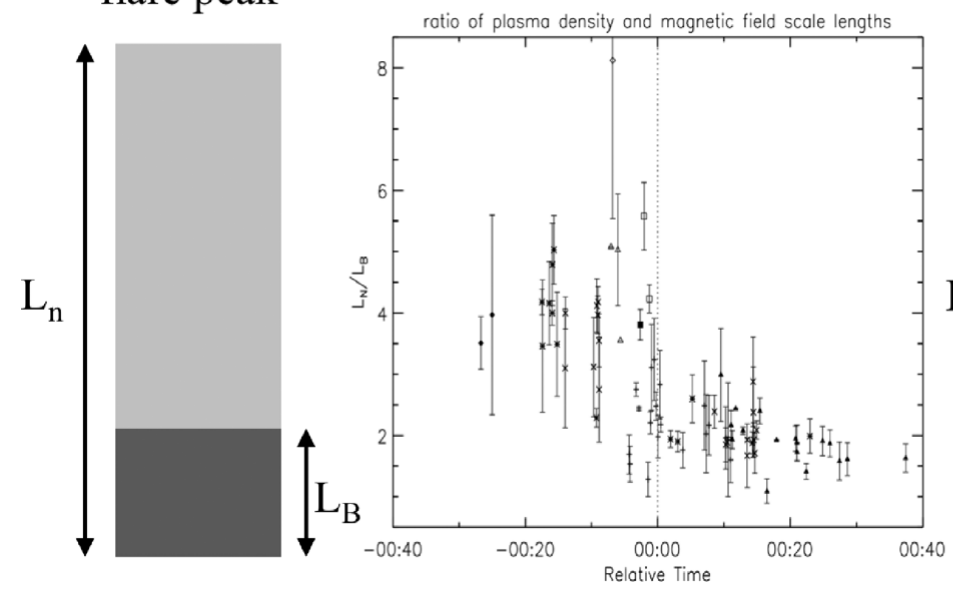

After the flare peak

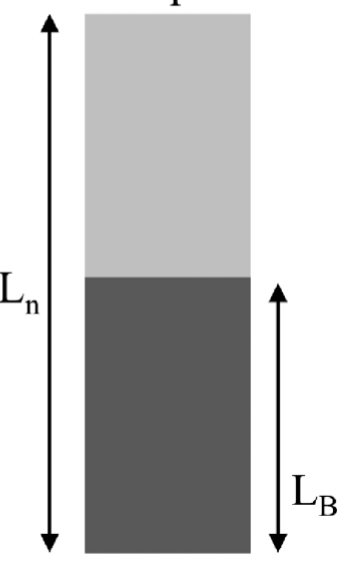

Figure 1. $L_{N} / L_{B}$ estimated from 74 microwave ZPs in the 9 flare events listed in Table 1, are plotted against the time relative to the GOES soft X-ray flaring peaks (vertical dotted line) of each flares. The symbols used to denote the ratio $L_{N} / L_{B}$ are also listed in Table 1 (Yu et al. 2012).

of between 1.5 and 5 . Before the flaring peak, the ratio is mainly in range of $3-5$, and after the peak the ratio decreases to $1-3$, which indicates that $L_{B}$ increases faster relative to the $L_{N}$ (cartoon in Fig.1). (2) The smaller these absolute values $L_{N}$ and $L_{B}$ are, the more stripes can be realized in certain frequency range. The absolute values of $L_{N}$ and $L_{B}$ varied in different events as the numbers of zebra stripes varied (Tabel.1), whereas the ratio $L_{N} / L_{B}$ is mainly in the range of $1.5-5$, independent from the varied absolute values. (3) Among the listed 9 events, we found 3 typical flares that ZPs appeared in both of before and after the soft X-ray flaring peaks. The ratio displays a step-wise decrease during the processes of topological changes of magnetic fields in flaring regions.

\section{Conclusions}

(1) The magnetic field scale height increases faster relative to the plasma density scale height during the flaring process.

(2) The $L_{N} / L_{B}$ variation is due to the topological change of flare core region where the main part of the magnetic energy is released.

\section{Acknowledgements}

This work is supported by MOST Grant No.2011CB811401, NFSC Grant No.10921303, 11273030, and the National Major Scientific Equipment R\&D Project ZDYZ2009-3.

\section{References}

Bastian, T. S., Benz, A. O., \& Gary, D. E. 1998, ARAA , 36, 131

Chernov, G. P. 2006, Space Sci. Revs, 127, 195

Elgaröy, O. 1959, Nature, 184, 887

Kuznetsov, A. A. \& Tsap, Y. T. 2007, Solar Phys., 241, 127

Tan, B. L., Yan, Y. H., Tan, C. M., Sych, R., \& Gao, G. N. 2012, ApJ, 744, 166

Yu, S. J., Yan, Y. H., \& Tan, B. L. 2012, ApJ, 761, 136 